\title{
ACOUSTICAL INVESTIGATIONS OF AMINO ACID IN AQUEOUS SACCHARIDE SOLUTION AT DIFFERENT TEMPERATURES
}

\author{
ARTI GUPTA $^{\mathrm{a} 1}$ AND ROLI SRIVASTAVA ${ }^{\mathrm{b}}$ \\ ${ }^{a b}$ Department of Chemistry, C.M.P.P.G. College, University of Allahabad, Prayagraj, U.P., India
}

\begin{abstract}
The density, ultrasonic velocity, viscosity of threonine with glucose and galactosehave been measured as a function of amino acid concentration atvarying temperatures. From these experimental data, various interaction parameters such as apparent molal volume, apparent molal adiabatic compressibility, relative association and relative viscosity has been calculated. The data has also been used to calculate limiting apparent molal volume, limitingapparent adiabatic compressibility, the corresponding constants $S_{v}$ and $S_{k}$. The viscosity $B$ coefficients have been calculated using Jones-Dole equation. Volumetric parameters indicate the interactions of saccharides with amino acid. These parameters have been interpreted in terms of solute-solute and solutesolvent interactions.
\end{abstract}

KEYWORDS: Ultrasonic Velocity, Viscosity, Density, Threonine, Saccharide

Volumetric, viscometric and ultrasonic velocity studies of amino acids in aqueous saccharide solutions can provide valuable information for understanding various fundamental processes like denaturation, folding-unfolding processes and stability of proteins due to solute-solvent and solute-solute interactions. Due to complex structure of proteins, direct investigation of the solute-solvent effect on these biological macromolecules is very challenging. Therefore, a useful approach is to study the amino acid which are the monomer units of proteins molecules. The interpretation of nature of amino acids is helpful in understanding the protein-saccharides interactions in solutions. Threonine is an essential genetically coded neutral $\alpha$-amino acid promotes normal growth, supports cardiovascular, liver, central nervous, and immune system function. It is required to help to maintain the proper protein balance in the body, as well as in the formation of collagen in the skin. Other nutrients are also better absorbed when threonine is present in the body. The study of saccharides has become a subject of increasing interest because of the multidimensional, physical, biochemical and industrially useful properties of these compounds (Chalikian, 2003; Santosh et al., 2009; Wang et al., 2006). In addition to their importance to the food, pharmaceutical and chemical industries, the simple saccharides have received considerable attention for their ability to protect biological macromolecules (Nikam et al., 2000; Banipal et al., 2007). The volumetric, viscometric, ultrasonic velocity and refractive index studies of amino acids in water and saccharides can provide valuable information for understanding protein unfolding and the hydrophobic interactions of non-polar side chains (Rajagopal et al., 2009; Enea et al., 1982; Perrin et al., 1980; Srivastava et al., 2010). Therefore, the study of saccharides-protein interactions is very important for immunology, biosynthesis, pharmacology and medicine. It is widely recognized that sugars help in stabilizing biological macromolecules. Since volumetric, compressibility and viscosity studies of amino acids are lacking in aqueous saccharides thus an attempt has been made to understand the behaviour of threonine in aqueous glucose and galactose through density, viscosity and ultrasonic velocity measurements at $303.15,308.15,313.15,318.15$ and $323.15 \mathrm{~K}$

\section{EXPERIMENTAL}

\section{MATERIALS AND METHODS}

Glucose (0.99) and galactose (0.98) extra pure A.R., Sisco Research Laboratories, India, were used after drying over anhydrous $\mathrm{CaCl}_{2}$ in a vacuum desiccators for 48 $\mathrm{h}$ at room temperature. All the chemicals used were purified by standard procedure, discussed by Perrin and Armarego (1980). All the samples were kept in tightly sealed bottles to minimize the absorption of atmospheric moisture. The solutions were prepared freshly by mass using an electronic balance (model GR-202R, Japan) with a precision of \pm 0.01 $\mathrm{mg}$ in doubly distilled deionized and degassed water. A double stem calibrated pyknometer and Ubbelohde type suspended level viscometer were used to determine the density and viscosity of solvent and solutions. The ultrasonic velocity of pure components and their mixtures

${ }^{1}$ Corresponding author 
were measured by fixed frequency interferometer of $2 \mathrm{MHz}$ (Mittal Enterprises, Model-05). The calibration of ultrasonic interferometer was done by measuring the velocity in A.R. grade benzene and carbon tetrachloride. Standard value of ultrasonic velocity for benzene and carbon tetrachloride were calculated from the literature value at different temperature (Lide, 1995). The measured values of ultrasonic velocity agree closely with the literature values. The maximum estimated error in ultrasonic velocity measurements has been found to be $\pm 0.1 \mathrm{~ms}^{-1}$. The temperature of the test liquids during the measurements was maintained by circulating water from an electronically controlled thermostatic water bath (JULABO, model ME-3, M/s Mittal Enterprises) covered with cotton jacket to avoid thermal dissipation. The viscosity was measured by Ubbelhode (Stokes et al., 1965) type suspended level viscometer with a water circulation jacket. At least four time flow measurements were performed for each composition and temperature, and the results were average. The viscometer was kept vertically in a transparent walled bath about $30 \mathrm{~min}$ to attain thermal equilibrium. The times of flow were recorded with a digital stopwatch with an accuracy of \pm 0.01 s. The viscosity data were reproducible within $\pm 0.001 \mathrm{Nsm}^{-2}$.

The temperature of the test liquids in every experiment of density, viscosity and ultrasonic velocity was maintained by circulating water through the jacket from an electronically controlled thermostatic water bath. A thermostatically controlled well-stirred water bath whose temperature was controlled to $\pm 0.01 \mathrm{~K}$ was used for the density, viscosity and sound velocity measurements. Fresh solutions in water have been prepared by the variation of stock solution of carbohydrates and threonine keeping the total volume constant in air tight stopper volumetric flasks. Mixtures were prepared by weighing the liquids in specially designed ground glass stopped bottles, taking extreme precautions to minimize preferential evaporation.

\section{THEORY AND CALCULATIONS}

The following acoustical and thermo dynamical parameters have been calculated using the standard relations.

Adiabatic compressibility $\beta=1 / \mathrm{U}^{2} \rho$

The apparent molal compressibility has been calculated from the relation, $\varphi_{\mathrm{k}}=\stackrel{1000}{\mathrm{~m} \rho 0}(\rho 0 \beta-\rho \beta 0)+\left(\begin{array}{c}\beta 0 \mathrm{M} \\ ---------)\end{array}\right.$

where $\beta, \rho$ and $\beta 0, \rho 0$ are the adiabatic compressibility and density of solution and solvent respectively, $\mathrm{m}$ is the molal concentration of the solute, and $\mathrm{M}$ is the molecular mass of the solute.

$\varphi \mathrm{k}=\varphi_{\mathrm{k}}^{0}+\mathrm{S}_{\mathrm{k}} \mathrm{m}^{0.5}$

where $\varphi_{\mathrm{k}}^{0}$ is the limiting apparent molal compressibility at infinite dilution and $\mathrm{S}_{\mathrm{K}}$ is a constant. $\varphi \mathrm{k}$ and $S_{K}$ of equation(3) have been evaluated by least square method. The apparent molal volume $\varphi_{\mathrm{V}}$ has been calculated using the relation,

\begin{tabular}{cc}
$M$ & $1000(\rho-\rho 0)$ \\
$\varphi_{\mathrm{v}}=(---)$ & - \\
\hline$-\mathrm{m} \rho \rho 0$
\end{tabular}

The apparent molal volume $\varphi_{\mathrm{V}}$ has been found to differ with concentration according to Masson's empirical relation as

$\varphi_{\mathrm{v}}=\varphi_{\mathrm{v}}^{0}+\mathrm{S}_{\mathrm{v}} \mathrm{m}^{0.5}$

where $\varphi^{0}$ is the limiting apparent molal volume at infinite dilution, $\mathrm{m}$ is the molal concentration of the solute and $S_{V}$ is a constant and these values were determined by least square method. The viscosity A and B coefficients of threonine in aqueous disaccharide solutions were calculated from the Jones-Dole equation (Jones et. al,1929).

$\eta / \eta_{0}=1+\mathrm{AC}^{0.5}+\mathrm{BC}$

where, $\eta$ and $\eta_{0}$ are the viscosities of the solution and solvent respectively. $\mathrm{A}$ is determined by the ionic attraction theory of Falkenhagen-Vernon and therefore also called Falkenhagen coefficient, B or Jones-Dole coefficient is an empirical constant determined by ion solvent interactions. The molal hydration number has been computed using the equation,

Molal hydration number $\mathrm{n}_{\mathrm{H}}=\underset{\mathrm{n}_{2}}{(-----)}\left(\begin{array}{cc}\mathrm{n}_{1} & \beta \\ \mathrm{n}_{2} & \beta_{0}\end{array}\right.$

Molar refraction, $\mathrm{R}_{\mathrm{m}}=\left(\mathrm{n}^{2}-1 / \mathrm{n}^{2}+2\right) \times \mathrm{V}_{\mathrm{m}}$

Rao's molar function $\quad \mathrm{R}=\mathrm{M}\left(\mathrm{u}^{1 / 3}\right) / \rho$

Solvation no. $=\mathrm{M}_{2} /\left(\mathrm{M}_{1}\left(1-\left(\beta_{\mathrm{s}} / \beta_{0}\right)(100-\mathrm{X}) / \mathrm{X}\right)\right.$ 
Redlich- kister eqn. $\mathrm{Y}^{\mathrm{E}=} \mathrm{X}_{1}\left(1-\mathrm{X}_{1}\right) \sum \mathrm{A}_{\mathrm{k}}\left(2 \mathrm{X}_{1}-1\right)^{\mathrm{k}}$

$\mathrm{Y}^{\mathrm{E}}=\mathrm{Yexp}-\mathrm{Yid}$

where $\mathrm{k}$ is the number of estimated parameters and $A_{K}$, the polynomial coefficients were obtained by fitting the equation to the experimental results by least-squares regression method and $\mathrm{Y}$ may be any calculated physical parameter. An excess property of a solution is defined as the difference between the actual mixture property and that which would be obtained for an ideal solution at the same temperature, pressure and composition. So the excess molar properties represent the deviation from ideal behavior of the mixtures. The excess functions are found to be very sensitive towards mutual interactions between the component molecules of the binary mixtures. The sign and the extent of deviation of the functions from ideality depend on the strength of interactions between unlike molecules. In order to study the non-ideality of the liquid mixtures excess parameters $\left(\mathrm{Y}^{\mathrm{E}}\right)$ of all the acoustic parameter were computed where $\mathrm{X}_{1}$ is the mole fraction of the amino acid $\mathrm{n}_{1}=$ no. of moles of solvent, $\mathrm{n}_{2}=$ no. of moles of solute, $\mathrm{M}_{\text {eff }}=\mathrm{M}_{1} \mathrm{~W}_{1}+\mathrm{M}_{2} \mathrm{~W}_{2}$, where $\mathrm{M}$ is effective molecular weight, 'b' stands for cubic packing assumed to be 2 for liquids and ' $\mathrm{K}$ ' is a dimensionless constant independent of temperature and nature of liquids and its value is $4.281 \times 10^{9}$, $\mathrm{T}$ is the absolute temperature, and $\mathrm{R}$ is gas constant.

\section{RESULTS AND DISCUSSION}

The study of amino acids in aqueous saccharides has been analysed at different temperatures. In aqueous solutions, amino acids behave as zwitterions, their hydration and interaction with proteins showresemblances (Ali et al., 2007; Bamezai et al., 2011; Kannappana et al., 2007). Thermodynamic properties show the interactions occurring between amino acids and other components (Burakowski et al., 2010; Fort et al., 1965). In this paper, acoustical studies of threonine in aqueous saccharides solutions is presented. The ultrasonic velocity data as such do not provide significant information about the relative strength of various types of interionic or intermolecular interactions between the components. Thus, an attempt has been made to understand the interactions between threonine and saccharides in aqueous medium using density, viscosity and ultrasonic velocity measurements at $303.15,308.15$, $313.15,318.15$ and $323.15 \mathrm{~K}$.
The experimental data of density, viscosity and ultrasonic velocity for threonine and glucose as system (i) and threonine and galactose as system (ii) at different temperatures are reported in Table (1-3). Various physical and thermodynamical parameters like hydration number $\left(\mathrm{n}_{\mathrm{H}}\right)$, adiabatic compressibility ( $\left.\beta \mathrm{s}\right)$, apparent molar compressibility $\left(\varphi_{\mathrm{k}}\right)$ and their constants $\left(\mathrm{S}_{\mathrm{K}}, \mathrm{S}_{\mathrm{V}}\right)$, viscosity $\mathrm{A}$ and $\mathrm{B}$ coefficients of Jones-Dole equation were calculated from the density, viscosity and ultrasonic velocity data and the results are presented in Tables (4-7). Parameters are discussed in terms of solute-co-solute and solute-solvent interactions. Excess properties are fitted to Redlich-kisterpolynomial equation. The coefficients $\mathrm{A}_{0}, \mathrm{~A}_{1}$, $\mathrm{A}_{2}$ and standard deviation are calculated (Table 8).

Table (1) reveals that the values of density increases with increase in molal concentration of threonine. This increasing trend of the density suggests that there is a moderate strong electrolytic nature in which the solute tends to attract the solvent molecules. The increase in the value of density may be attributed to the increase in hydrophilic interactions. The values of the effect of viscosity and ultrasonic velocity, summarized in tables (2) and (3). The data show that the increase in the ultrasonic velocity is due to the cohesion brought about by ionic hydration. When threonine is dissolved in water the positive and negative ions are formed. The water molecules are attached to these ions by electrostatic forces thereby increasing greater cohesion in solution. The electrostriction effect which brings about the shrinkage in the volume of solvent caused by zwitterion portion of solute is increased in solvent in comparison to that of water. This shows that the system under consideration behaves like structure maker.

Table (4) shows that the values of $n_{H}$ are positive in the systems studied and positive values of hydration indicate an appreciable solvation of solutes. These values of $\mathrm{n}_{\mathrm{H}}$ are found to increase with increasing threonine content in the systems studied. This supports the structure promoting nature of the solutes and the presence of a appreciable dipole-dipole interaction between solute and water molecules. This also suggests that compressibility of the solution will be less than that of the solvent. As a result, solutes will gain mobility and have more probability of contacting solvent molecules. This may enhance the interaction between solute and solvent molecules. 
Table (5) shows that the structural arrangement of molecule results in decreasing adiabatic compressibility by showing intermolecular interactions (Burakowski et al., 2010). The decrease in compressibility imply that there is enhanced molecular associations in these system on increase in solute content, as the new entities formed, become compact and less compressible (Fort et al., 1965). There is decrease in inter-ionic interactions with increase in temperatures may be due to more solvation of solute ions with rise in temperatures. Thus structural arrangement of molecular results in decreasing adiabatic compressibility by showing intermolecular interactions is also reported. There is decrease in inter-ionic interactions with increase in temperatures and this is may be due to more solvation of solute ions. Amino acid molecules of neutral solution exist in the dipolar form and thus have stronger interaction with the surrounding water molecules. The increasing electrostrictive compression of water around the molecules results in a large decrease in the compressibility of solutions. Hydrophilic-ionic interactions between the hydroxyl group of saccharides and zwitterionic centres of the amino acids. The hydrophilic-hydrophobic interactions between the hydroxyl group of the saccharide molecules and the non-polar side group of the amino acids. Hydrophilic-hydrophilic interactions between the hydroxyl group of the saccharide molecules and hydroxyl group of the amino acids hydrophobic-hydrophobic interactions between the non-polar side groups of the saccharide molecules and the amino acids.
Table (6) implies that the molar compressibility $\left(\varphi_{\mathrm{k}}\right)$ values of the amino acids in aqueous saccharides solution indicate the presence of solute-solvent interactions showing the hydrophilic and ionic interactions occurring in the systems. More number of water molecules is present at lower concentration of saccharides, thus the chances for the penetration of solute molecules into the solvent molecules is more. This behavior of $\varphi_{\mathrm{k}}$ indicates the strengthening of the solute-solvent interaction in the systems.

For the entire composition range of aqueous saccharides at different temperatures, the values of the A coefficient indicating the presence of solute-solute interactions (Table 7). The A coefficient values may indicate the presence of very weak ionic interactions in the solution. The B-coefficient value is a measure of the effective solvodynamic volume of solvated ions and is governed by ion-solvent interactions. The solvent molecules have to be wrenched out of the bulk solvent. Excess properties are fitted to Redlich-kisterpolynomial equation and coefficients, $\mathrm{Ai}$ and evaluated by using Redlich-kister polynomial equation by the method of leastsquares. The coefficients $\mathrm{A}_{0}, \mathrm{~A}_{1}, \mathrm{~A}_{2}$ and standard deviation are calculated (Table 8). The observed trend indicates the presence of specific interaction between the solute and the solvent. This is due to intermolecular hydrogen bonding and it is specific dipolar interaction in proton atoms and non bonding electrons.

Table 1: Density ( $\rho)$ of systems at different temperature (K)

\begin{tabular}{|c|c|c|c|c|c|}
\hline $\mathrm{C}\left(\mathrm{mol}_{\mathrm{m}}^{-3}\right) / \mathrm{T}(\mathrm{K})$ & 303.15 & 308.15 & 313.15 & 318.15 & 323.15 \\
\hline System (i) & \multicolumn{5}{|c|}{$\rho \times 10^{-2}\left(\mathrm{~kg} \mathrm{~m}^{-3}\right)$} \\
\hline 0 & 9.956 & 9.940 & 9.922 & 9.902 & 9.880 \\
\hline 10.0 & 9.971 & 9.946 & 9.945 & 9.921 & 9.904 \\
\hline 12.5 & 9.985 & 9.966 & 9.953 & 9.939 & 9.924 \\
\hline 16.5 & 9.988 & 9.967 & 9.959 & 9.952 & 9.928 \\
\hline 25.0 & 9.997 & 9.977 & 9.977 & 9.976 & 9.931 \\
\hline 50.0 & 10.011 & 9.980 & 9.979 & 9.978 & 9.976 \\
\hline 100.0 & 10.139 & 10.074 & 9.999 & 9.996 & 9.981 \\
\hline 200.0 & 10.164 & 10.150 & 10.051 & 10.049 & 10.028 \\
\hline System (ii) & & & & & \\
\hline 10.0 & 9.967 & 9.896 & 9.867 & 9.866 & 9.857 \\
\hline 12.5 & 9.968 & 9.897 & 9.868 & 9.867 & 9.858 \\
\hline 16.5 & 9.977 & 9.932 & 9.868 & 9.866 & 9.863 \\
\hline 25.0 & 9.978 & 9.935 & 9.878 & 9.868 & 9.867 \\
\hline 50.0 & 9.981 & 9.936 & 9.881 & 9.873 & 9.867 \\
\hline 100.0 & 9.991 & 9.937 & 9.889 & 9.885 & 9.870 \\
\hline 200.0 & 10.012 & 9.953 & 9.922 & 9.892 & 9.880 \\
\hline
\end{tabular}




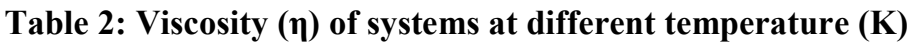

\begin{tabular}{|c|c|c|c|c|c|}
\hline $\mathrm{C}\left(\mathrm{mol} . \mathrm{m}^{-3}\right) / \mathrm{T}(\mathrm{K})$ & 303.15 & 308.15 & 313.15 & 318.15 & 323.15 \\
\hline System (i) & \multicolumn{5}{|c|}{$\eta \times 10^{-2}\left(\mathrm{kgm}^{-1} \mathrm{~s}^{1}\right)$} \\
\hline 0 & 8.007 & 7.225 & 6.560 & 5.986 & 5.494 \\
\hline 10.0 & 6.731 & 6.287 & 5.910 & 5.642 & 5.305 \\
\hline 12.5 & 7.311 & 6.947 & 6.024 & 6.003 & 5.847 \\
\hline 16.5 & 7.366 & 6.990 & 6.041 & 6.003 & 5.887 \\
\hline 25.0 & 8.271 & 7.447 & 7.342 & 6.678 & 6.619 \\
\hline 50.0 & 8.295 & 7.843 & 7.786 & 7.304 & 6.708 \\
\hline 100.0 & 8.649 & 8.156 & 8.116 & 7.455 & 7.168 \\
\hline 200.0 & 8.823 & 8.443 & 8.312 & 7.750 & 7.352 \\
\hline \multicolumn{6}{|l|}{ System (ii) } \\
\hline 10.0 & 6.905 & 6.280 & 5.883 & 5.262 & 4.778 \\
\hline 12.5 & 6.905 & 6.280 & 5.883 & 5.262 & 4.778 \\
\hline 16.5 & 6.916 & 6.309 & 5.859 & 5.252 & 4.777 \\
\hline 25.0 & 6.920 & 6.325 & 5.907 & 5.410 & 4.820 \\
\hline 50.0 & 6.962 & 6.364 & 5.932 & 5.334 & 4.746 \\
\hline 100.0 & 6.777 & 6.407 & 5.976 & 5.370 & 4.866 \\
\hline 200.0 & 7.019 & 6.480 & 6.095 & 5.444 & 4.945 \\
\hline
\end{tabular}

Table 3: Ultrasonic velocity (u) of systems at different temperature (K)

\begin{tabular}{|c|c|c|c|c|c|}
\hline $\mathrm{C}\left(\mathrm{mol} . \mathrm{m}^{-3}\right) / \mathrm{T}(\mathrm{K})$ & 303.15 & 308.15 & 313.15 & 318.15 & 323.15 \\
\hline System (i) & \multicolumn{5}{|c|}{$\mathrm{u} \times 10^{-2}\left(\mathrm{~ms}^{-1}\right)$} \\
\hline 0.0 & 1509.5 & 1516.8 & 1528.4 & 1535.6 & 1541.4 \\
\hline 10.0 & 1338.0 & 1326.8 & 1324.5 & 1214.0 & 1210.8 \\
\hline 12.5 & 1348.0 & 1336.8 & 1334.5 & 1224.0 & 1220.8 \\
\hline 16.5 & 1354.8 & 1351.8 & 1351.8 & 1331.7 & 1312.0 \\
\hline 25.0 & 1357.8 & 1354.3 & 1352.8 & 1338.9 & 1313.0 \\
\hline 50.0 & 1367.8 & 1364.3 & 1362.8 & 1348.9 & 1323.0 \\
\hline 100.0 & 1377.8 & 1374.3 & 1372.8 & 1358.9 & 1333.0 \\
\hline 200.0 & 1387.8 & 1384.3 & 1382.8 & 1368.9 & 1343.0 \\
\hline \multicolumn{6}{|l|}{ System (ii) } \\
\hline 10.0 & 1353.0 & 1345.5 & 1345.0 & 1343.8 & 1329.0 \\
\hline 12.5 & 1353.0 & 1345.5 & 1345.0 & 1343.8 & 1329.0 \\
\hline 16.5 & 1363.0 & 1355.5 & 1355.0 & 1353.8 & 1339.0 \\
\hline 25.0 & 1373.0 & 1365.5 & 1365.0 & 1363.8 & 1361.5 \\
\hline 50.0 & 1383.0 & 1375.5 & 1375.0 & 1373.8 & 1371.5 \\
\hline 100.0 & 1393.0 & 1385.5 & 1385.0 & 1383.8 & 1381.5 \\
\hline 200.0 & 1403.0 & 1395.5 & 1395.0 & 1393.8 & 1391.5 \\
\hline
\end{tabular}


Table 4: Hydration number $\left(\mathrm{n}_{\mathrm{H}}\right)$ of systems at different temperature $(\mathrm{K})$

\begin{tabular}{|c|c|c|c|c|c|}
\hline $\mathrm{C}\left(\mathrm{mol}^{-3} \mathrm{~m}^{-3} / \mathrm{T}(\mathrm{K})\right.$ & 303.15 & 308.15 & 313.15 & 318.15 & 323.15 \\
\hline System (i) & \multicolumn{5}{|c|}{$\mathrm{n}_{\mathrm{H}} \mathrm{x} 0^{2}$} \\
\hline 10.0 & 0.858 & 0.986 & 1.170 & 0.719 & 0.976 \\
\hline 12.5 & 0.920 & 1.050 & 1.231 & 0.787 & 1.044 \\
\hline 16.5 & 0.970 & 1.136 & 1.327 & 1.299 & 1.457 \\
\hline 25.0 & 1.014 & 1.182 & 1.373 & 1.372 & 1.503 \\
\hline 50.0 & 1.198 & 1.380 & 1.592 & 1.591 & 1.750 \\
\hline 100.0 & 1.373 & 1.550 & 1.748 & 1.746 & 1.910 \\
\hline 200.0 & 1.432 & 1.623 & 1.810 & 1.809 & 1.968 \\
\hline System (ii) & & & & & \\
\hline 10.0 & 0.910 & 1.034 & 1.213 & 1.254 & 1.425 \\
\hline 12.5 & 0.964 & 1.091 & 1.268 & 1.314 & 1.485 \\
\hline 16.5 & 1.020 & 1.153 & 1.320 & 1.366 & 1.581 \\
\hline 25.0 & 1.124 & 1.264 & 1.439 & 1.485 & 1.710 \\
\hline 50.0 & 1.219 & 1.362 & 1.544 & 1.591 & 1.822 \\
\hline 100.0 & 1.449 & 1.608 & 1.815 & 1.870 & 2.127 \\
\hline 200.0 & 1.505 & 1.660 & 1.869 & 1.915 & 2.169 \\
\hline $\mathrm{C}\left(\mathrm{mol} . \mathrm{m}^{-3}\right) / \mathrm{T}(\mathrm{K})$ & 303.15 & 308.15 & 313.15 & 318.15 & 323.15 \\
\hline
\end{tabular}

Table 5: Adiabatic compressibility $\left(\beta_{\mathrm{s}}\right)$ of systems at different temperature $(\mathrm{K})$

\begin{tabular}{|c|c|c|c|c|c|}
\hline $\mathrm{C}\left(\mathrm{mol} . \mathrm{m}^{-3}\right) / \mathrm{T}(\mathrm{K})$ & 303.15 & 308.15 & 313.15 & 318.15 & 323.15 \\
\hline System (i) & \multicolumn{5}{|c|}{$\beta_{\mathrm{s}} \times 10^{10}\left(\mathrm{~kg}^{-1} \mathrm{~ms}^{2}\right)$} \\
\hline 10.0 & 5.602 & 5.712 & 5.732 & 6.839 & 6.888 \\
\hline 12.5 & 5.511 & 5.615 & 5.641 & 6.716 & 6.762 \\
\hline 16.5 & 5.455 & 5.491 & 5.495 & 5.666 & 5.851 \\
\hline 50.0 & 5.340 & 5.384 & 5.396 & 5.508 & 5.727 \\
\hline 100.0 & 5.196 & 5.256 & 5.307 & 5.418 & 5.639 \\
\hline 200.0 & 5.109 & 5.142 & 5.203 & 5.311 & 5.529 \\
\hline System (ii) & & & & & \\
\hline 10.0 & 5.481 & 5.586 & 5.602 & 5.626 & 5.758 \\
\hline 12.5 & 5.401 & 5.500 & 5.520 & 5.531 & 5.658 \\
\hline 16.5 & 5.317 & 5.400 & 5.439 & 5.450 & 5.470 \\
\hline 25.0 & 5.240 & 5.320 & 5.355 & 5.370 & 5.388 \\
\hline 50.0 & 5.163 & 5.243 & 5.276 & 5.290 & 5.310 \\
\hline 100.0 & 5.085 & 5.168 & 5.196 & 5.208 & 5.233 \\
\hline 200.0 & 5.003 & 5.086 & 5.106 & 5.130 & 5.153 \\
\hline
\end{tabular}


Table 6: $\varphi_{\mathrm{k}}$ of system at different temperature $(\mathrm{K})$

\begin{tabular}{|c|c|c|c|c|c|}
\hline $\mathrm{C}\left(\mathrm{mol}_{\mathrm{m}}^{-3}\right) / \mathrm{T}(\mathrm{K})$ & 303.15 & 308.15 & 313.15 & 318.15 & 323.15 \\
\hline System (i) & \multicolumn{5}{|c|}{$\varphi_{\mathrm{k}} \times 10^{10}$} \\
\hline 10.0 & 11.73 & 13.44 & 20.61 & 0.83 & 10.65 \\
\hline 12.5 & 26.47 & 30.10 & 43.96 & 5.83 & 25.39 \\
\hline 16.5 & 42.39 & 51.64 & 73.65 & 70.28 & 90.89 \\
\hline 25.0 & 59.57 & 71.79 & 101.20 & 101.41 & 122.96 \\
\hline 50.0 & 83.75 & 97.80 & 134.58 & 135.06 & 167.80 \\
\hline 100.0 & 116.29 & 127.92 & 159.11 & 159.72 & 194.58 \\
\hline 200.0 & 123.25 & 143.08 & 172.03 & 173.58 & 207.15 \\
\hline System (ii) & & & & & \\
\hline 10.0 & -116.33 & -117.69 & -120.65 & -161.09 & -180.25 \\
\hline 12.5 & -77.75 & -79.06 & -80.02 & -107.32 & -119.74 \\
\hline 16.5 & -33.27 & -34.62 & -32.83 & -43.40 & -51.55 \\
\hline 25.0 & 23.43 & 25.38 & 25.81 & 32.97 & 38.71 \\
\hline 50.0 & 86.32 & 91.59 & 91.17 & 115.32 & 134.39 \\
\hline 100.0 & 124.99 & 131.03 & 131.10 & 164.18 & 188.86 \\
\hline 200.0 & 133.90 & 139.64 & 141.59 & 171.86 & 197.03 \\
\hline
\end{tabular}

Table 7: Various constants for systems at different temperature (K)

\begin{tabular}{|c|c|c|c|c|c|}
\hline $\mathrm{C}\left(\mathrm{mol.m}^{-3}\right) / \mathrm{T}(\mathrm{K})$ & 303.15 & 308.15 & 313.15 & 318.15 & 323.15 \\
\hline & \multicolumn{5}{|c|}{$\mathrm{Sv} \times 10^{5}$ (Masson's) } \\
\hline System (i) & -5.072 & -3.322 & -1.132 & -0.475 & -1.589 \\
\hline System (ii) & -2.214 & 12.830 & 17.659 & 14.737 & 10.557 \\
\hline & $\varphi_{\mathrm{k}}^{0} \times 10^{10}$ & & & & \\
\hline System (i) & 10.010 & 11.303 & 12.851 & 15.012 & 16.910 \\
\hline System (ii) & 34.332 & 35.510 & 35.873 & 45.289 & 51.600 \\
\hline & $\mathrm{S}_{\mathrm{k}} \times 10^{9}$ & & & & \\
\hline System (i) & -0.096 & 0.069 & 1.450 & -0.835 & 0.358 \\
\hline System (ii) & -12.552 & -12.838 & -12.993 & -16.756 & -18.936 \\
\hline & $\begin{array}{c}\mathrm{B} \times 10^{4}(\text { Jones- } \\
\text { Dole) }\end{array}$ & & & & \\
\hline System (i) & 34.734 & 27.742 & 34.784 & 22.675 & 6.721 \\
\hline System (ii) & 76.092 & 74.149 & 61.791 & 66.248 & 76.422 \\
\hline & $\begin{array}{c}\text { A ( Jones-Dole) } \\
\text { Falkenhagen } \\
\text { coefficient }\end{array}$ & & & & \\
\hline System (i) & 3.473 & 2.774 & 3.478 & 2.268 & 0.672 \\
\hline System (ii) & 7.609 & 7.415 & 6.179 & 6.625 & 7.642 \\
\hline C(mol.m $\left.{ }^{-3}\right) / \mathrm{T}(\mathrm{K})$ & 303.15 & 308.15 & 313.15 & 318.15 & 323.15 \\
\hline
\end{tabular}


Table 8: Redlich-Kister coefficients for second order polynomial and standard deviation for various properties for systems at $303.15 \mathrm{~K}$

\begin{tabular}{|c|c|c|c|c|}
\hline & $\mathrm{A}_{2}$ & $\mathrm{~A} 1$ & $\mathrm{~A}_{0}$ & $\Sigma$ \\
\hline $\mathrm{u}\left(\mathrm{ms}^{-1}\right)$ & \multicolumn{5}{|l|}{} \\
\hline System (i) & $4.406 \mathrm{E}+04$ & $3.561 \mathrm{E}+03$ & $1.405 \mathrm{E}+03$ & 8.545 \\
\hline System (ii) & $-1.060 \mathrm{E}+04$ & $-5.859 \mathrm{E}+02$ & $1.386 \mathrm{E}+03$ & 21.604 \\
\hline$\rho\left(\mathrm{kgm}^{-3}\right)$ & & & & \\
\hline System (i) & $2.353 \mathrm{E}+04$ & $1.708 \mathrm{E}+03$ & $1.023 \mathrm{E}+03$ & 6.496 \\
\hline System (ii) & $-7.702 \mathrm{E}+02$ & $-3.294 \mathrm{E}+01$ & $9.985 \mathrm{E}+02$ & 1.710 \\
\hline$\eta\left(\mathrm{kgm}^{-1} \mathrm{~s}^{-1}\right)$ & & & & \\
\hline System (i) & $1.899 \mathrm{E}+05$ & $1.589 \mathrm{E}+04$ & $9.896 \mathrm{E}+02$ & 26.791 \\
\hline System (ii) & $-7.037 \mathrm{E}+02$ & $-7.121 \mathrm{E}+01$ & $6.940 \mathrm{E}+02$ & 11.470 \\
\hline
\end{tabular}

\section{CONCLUSION}

Ultrasonic velocity, density and viscosity of threonine in aqueous saccharides solution as well as in varying composition have determined.. Using these quantities, various parameters adiabatic compressibility, apparent molal volume, apparent molal compressibility, relative association, viscosity B-coefficient are determined. These parameters have been correlated to the various types of interaction taking place in the system. The experimental results reveal the existence of ion-solvent interactions resulting in attractive forces promote the structure making tendency while solute-solute interactions resulting in dipole-dipole, dipole-induced dipole and electrostrictive forces enhance the structure breaking properties of amino acid. This suggests theronine in aqueous saccharide mixture is a strong structure maker.

\section{ACKNOWLEDGEMENT}

Authors are thankful to U.G.C., India for providing financial assistance.

\section{REFERENCES}

Ali A., Khan S. and Nabi F., 2007. Volumetric, viscometric and refractive index behaviour of amino acids in aqueous glycerol at different temperatures. J. Serb. Chem. Soc., 72(5): 495-512.

Bamezai R.K. and Anjana, 2011. Ultrasonic and volumetric investigations of L-threonine in aqueousand in mixed aqueous solution, Scholars Research
LibraryArchives of Applied Science Research, 3(1):370-379.

Banipal T.S., Kaur D., Banipal P.K. and Singh G., 2007. Thermodynamic and transport properties of 1serine and 1-threonine in aqueous sodium acetate and magnesium acetate solutions at T $298.15 \mathrm{~K}$. J. Chem. Thermodynamics, 39: 371-384.

Burakowski A. and Gliński J., 2010. Hydration of Amino Acids from Ultrasonic Measurements. J. Phys. Chem. B., 114(37):12157-12161.

Chalikian T.V., 2003. Volumetric properties of proteins. Annu. Rev Biophys. Biomol. Struct., 32: 207.

Enea O. and Jolicoeur C., 1982. Ultrasonic and volumetric investigations of L-threonine in aqueous and in mixed aqueous solution. J. Phys. Chem., 86: 3870.

Fort R.J. and Moore W.R., 1965. Adiabatic compressibilities in binary liquid mixtures, Trans Faraday. Soc., 61: 2102.

Jones G. and Dole M., 1929. Viscosities of aqueous solutions of strong electrolytes with special references to barium chloride. J. Am. Chem. Soc., 51:2950.

Kannappana A.N. and Palani R., 2007. Ultrasonic investigations in amino acids with aqueous dimethyl formamide. Ind. Nat. Sci. Acad., 46:5459. 
GUPTA AND SRIVASTAVA: ACOUSTICAL INVESTIGATIONS OF AMINO ACID IN AQUEOUS SACCHARIDE SOLUTION...

Lide D.R., 1995-1996. CRC Handbook of Chemistry and Physics, $76^{\text {th }}$ edition India: CRC Press.

Nikam P.S., Ansari H.R. and Hasan M., 2000. Acoustical properties of fructose and maltose solutions in water and in aqueous $0.5 \mathrm{M} \mathrm{NH}_{4} \mathrm{Cl}$. J. Mol. liq., 84:169-178.

Perrin D.D. and Armarego W.L.F., 1980. Purification of Laboratory Chemicals, Oxford, Pergamon Press.

Rajagopal K. and Jayabalakrishnan S.S., 2009. Volumetric and viscometric studies of 4-aminobutyric acid in aqueous solutions of salbutamol sulphate at 308.15, 313.15 and 318.15 K.Chin. J. Chem. Eng., 17: 796-804.

Santosh M.S., Bhat D.K. and Bhat A.S., 2009. Molecular interaction in glycylglycine- $\mathrm{MnCl}_{2}$ aqueous solution at $(288.15,293.15,298.15,303.15$, 308.15, 313.15 and 318.15) K.J. Chem. Eng., 54(10): 2813-8.

Srivastava R., Saksena A.R. and Gupta A., 2010. Viscometric studies on the molecular association of biomolecules based on thermodynamic parameters supported by UV spectra. Asian J. Appl. Sci., 3(1):13-24.

Stokes R.H. and Mills R., 1965. Viscosity of Electrolytes \& Related Properties, New York, NY: Pergamon Press.

Wang Y., Liu Q. and Zhuo K., 2006. Volumetric and viscosity properties of monosaccharides in aqueous amino acid solutions at $298.15 \mathrm{~K}$. J. Chem. Eng. Data, 51(3): 919-927. 